\title{
APPARENT SOLAR DIAMETER AND VARIABILITY
}

\author{
A. VIGOUROUX \\ Laboratoire G.D. Cassini, U.M.R. CNRS 6529 \\ Observatoire de la Côte d'Azur \\ B.P. 4229, 06304 Nice Cedex 04, France
}

\section{Introduction}

Philippe Delache brought me to solar physics in late June 1992. The first things he began with was the diameter of the Sun as it is measured at the "plateau de Calern", and my first work was to analyze this time series in order to separate the "noise" from the pertinent information (section 2). Wet then applied the technique developed for this purpose to the study of the 11-year solar cycle as seen in the Wolf sunspot number (section 3) and I continued in collaboration with Judit Pap with the study of total irradiance and some other solar indicators which could help to understand its variations (section 4).

\section{Study of the diameter}

\subsection{WHY DO WE STUDY THE DIAMETER OF THE SUN ?}

The diameter is a by-product of astrometric measurements: the best data are obtained from transit times through the meridian or through an elevation circle. The most homogeneous time series obtained from an astrolabe is due to F. Laclare from Observatoire de la Côte d'Azur, France (Laclare et al. 1996) but other measurements are available from astrolabes established in Belgrade (Ribes et al. 1988) or Brazil (Leister and Benevides-Soares 1990).

The diameter can also be a by-product of measurements of solar oblateness or more generally of the limb figure (obtained for example from solar images from space).

The ground-based measurements provide the necessary long-term time series whereas the satellite measurements may provide a way to separate 
possible variations induced by terrestrial atmosphere from real solar variations.

In $1983, F$. Laclare found that the diameter might vary in time (Laclare 1983). From this, Philippe Delache made many different studies in order to determine and understand the origin of the observed variations.

Measurements of the diameter and its possible variations are of prime importance since the present theory predicts no observable changes (Spruit 1994) whereas measurements do show variations (up to 0.1 ").

We can ask a few questions about the sources of such variations:

- what is the influence of the terrestrial atmosphere?

- is there any variations in the limb profile?

- is there any variation in the energy production?

- what are the magnetic fields and/or convection effects?

\subsection{THE TIME SERIES OF THE CERGA ASTROLABE}

Reliable and coherent results are obtained when observations are homogeneous (a single observer) and available over a long enough time span (at least a solar cycle): the CERGA astrolabe provides such time series from 1975 up to now.

The instrument is a Danjon-type astrolabe which has been adapted for solar observations. The single equilateral prism has been replaced by a set of 11 reflector prisms which allow observations at various zenith distances. Two images of the Sun are observed: a direct one and an other reflected off a level mercury surface. The procedure consists in timing the point at which the upper edge of the direct image is in contact with the inner edge of the reflected image. The second timing is proceeded when the reverse occurs. The radius is half the difference between the zenith distances computed at the times of the two crossings (Laclare et al. 1996) and is represented on figure 1a.

The differential refraction should not affect the measurements of the vertical diameter since the opposite sides of the Sun are measured at equal altitudes. However, the thickness of the atmosphere changes with the zenith distance. Laclare et al. (1996) have indeed underlined a relation between measurements of the diameter and altitude: the diameter decreases when the zenith distance increases whereas, as could be guessed, the standard deviation of the measurements increases with altitude. This had been also noticed by Philippe Delache who had used this relation to work out the error bars. Distinguishing East and West measurements, he has, for each year and for each zenith distance, calculated the deviation from the corresponding mean of each data set. Then, a mean deviation over all years is calculated for each zenith distance and for East and West observations. Then, he 

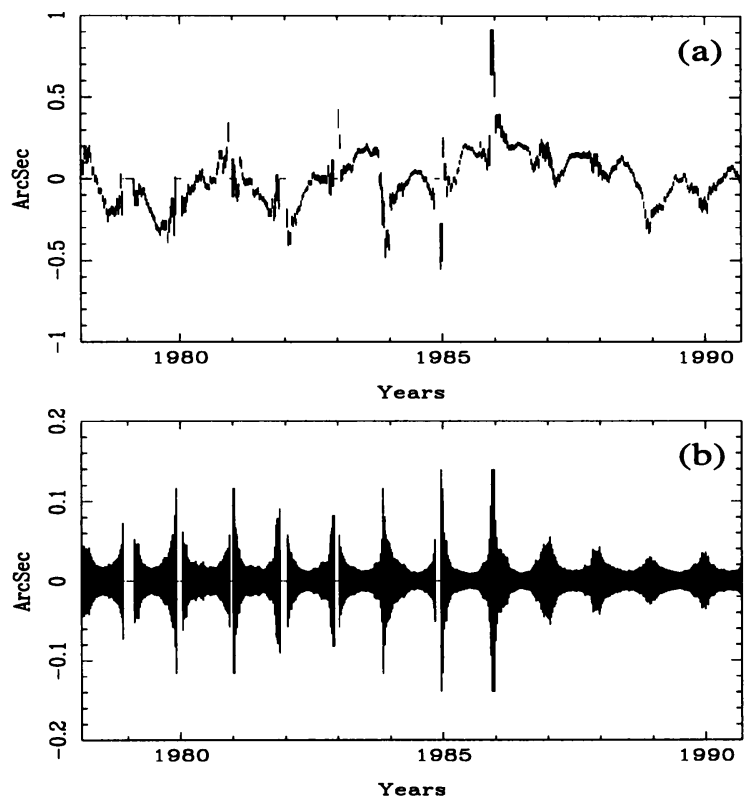

Figure 1. (a): Solar data observations: 1- $\sigma$ error bars.

(b): Time series of errors bars centered around zero. Notice the large variety of $\sigma_{i}$ 's amplitudes and the signature of seasons, the large errors bars corresponding to winters, especially in early years.

could assess an error bar to each individual measurement, given its zenith distance and direction of observation. Figure 1a shows 3-month average of the radius time series (only one point over nine is plotted) together with the corresponding error bars which are calculated as the reverse of the square root of the sum of the individual error bars within 3 month. Those mean error bars are represented on figure $1 \mathrm{~b}$ (being centering around 0 ): some error bars are larger, for example at the beginning of the time series where the number of individual measurements was not as high as it became later. They are also larger during winter, when observations are scarce, but also when the measurements are mostly obtained at larger zenith distance (low over the horizon) which increases the individual error bars of the data.

\subsection{HOW DO WE ASSESS THE REALITY OF OBSERVED VARIATIONS ?}

We can have at least three possibilities to find out whether the variations are solar in their origin or not:

- correlation between diameter and other solar time series

- sorting signal from noise, and assessing significance of model parameters such as periodicities or amplitudes 


\section{- compared polar and equatorial observations}

The first point has been investigated by Philippe Delache through correlations between the diameter and the Wolf sunspot number (Delache et al. 1985), the total solar irradiance (Delache et al. 1988, Delache, 1988), the p-mode frequencies and the neutrino flux (Delache et al. 1993, Gavryusev et al. 1994). The results show that the 11-year cycle of the radius variations is anti-correlated to the solar cycle observed in various indices but may be correlated with the neutrino flux variations.

We have investigated the second point developing methods using the wavelet analysis (Vigouroux and Delache 1993, see the following section).

Because of the Earth's orbital parameters, the vertical (horizontal) solar diameters observed from the Earth do not always correspond to the polar (equatorial) solar diameter. With its 11 prisms, F. Laclare can observe the heliographic latitudes from 15 degrees to 80 degrees. This has enabled a careful study of the radius variations with different inclinations. It has been first investigated by Philippe Delache (1988) and more recently by Laclare et al. (1996): the coherence is good between the radius variations at polar and equatorial inclinations (third point).

\subsection{ANALYSIS OF THE CERGA DIAMETER TIME SERIES}

Philippe Delache wanted to assess the reality of the observed variations of the Sun's radius. We therefore had to sort out the signal from the noise. The simplest analysis consists in making the Fourier transform of the data and cutting-off some of the high frequencies, and reconstructing the cleaned signal from the retained frequencies. This raises two problems:

- firstly: how can we determine in the Fourier plane the noise level ? In other words, are we sure that there is no signal in what we call noise?

- secondly: when we retain frequencies considered significant, are we sure that the corresponding standing oscillations (or waves) are present all along the time series? With the Fourier analysis, we cannot see if a frequency occurs here and there in the time series.

In order to answer the first question, we have studied in detail the nature (or origin) of uncertainties which constitute the so-called "error bars". We may then simulate the noise issued by the error bars, so that we are able to assess some significant cut-off level in the frequency domain. Simulating noisy data permits then to retain only the significant parts of actual signals.

To answer the second question, Philippe Delache proposed to make wavelet transform analysis. This can be viewed as a time and scale transform: it allows to detect structures at different scales (or within several frequency bands) and to locate them in time. Since the classical Fourier transform 


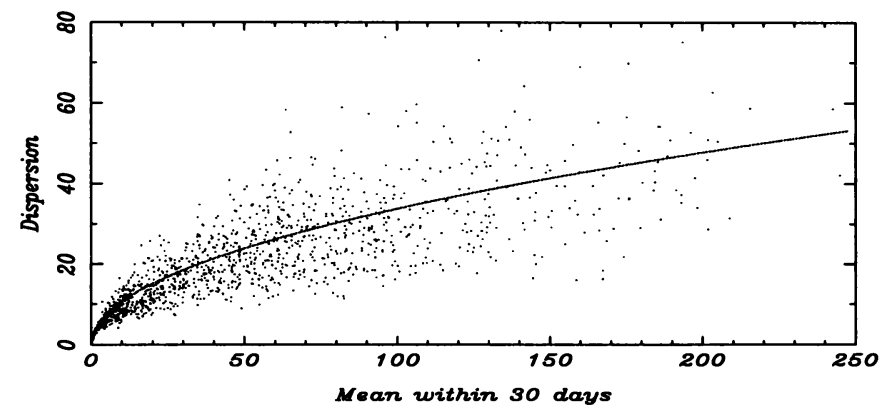

Figure 2. Dispersion of the daily value calculated within 30 days as a function of the corresponding mean. The parabolic fit is representative of a Poisson distribution of the daily Wolf number within 30 days.

gives only informations on frequency, the wavelets seem to be more appropriate for removing noise in a time series. Indeed, the noise may vary in time (as showed on figure 1b: the error bars are larger in winter than summer) and the Fourier techniques does not take into account its variations contrary to the wavelets.

We performed Fourier and wavelet analysis of the radius time series, assuming that the noise distribution within an error bar is Gaussian. The results obtained through both techniques are in favor of the wavelet analysis. Firstly because the wavelet reconstruction leaves out large error bars. Secondly the number of independent parameters needed for a reconstruction of the same quality is smaller in the wavelet analysis case: this latter representation is then simpler.

\section{Study of the Wolf number}

We then proposed to apply similar techniques to historical solar activity as measured by sunspot numbers. We still wanted to assess the degree of significance of the elements of Fourier or wavelet transforms of the data, taking care of their statistical properties. Here again, inverting the transform yields to "models" for the variation of initial data which retain only these significant elements.

The Wolf sunspot number contains no uncertainties, contrary to the radius time series. However, some of the day-to-day variations may not be completely deterministic. We used the monthly sunspot number as our time series to analyze and we proposed to associate to every monthly data the dispersion of the daily sunspot numbers that it includes, considering the dispersion as the relevant "uncertainty". The latter is however more solar than instrumental in its origin. Moreover, it appeared that the dispersion strongly depends upon the mean activity (figure 2). Actually a parabolic 
(a)

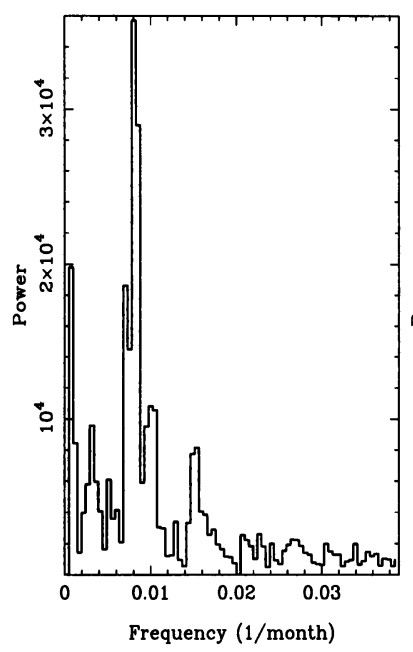

(b)

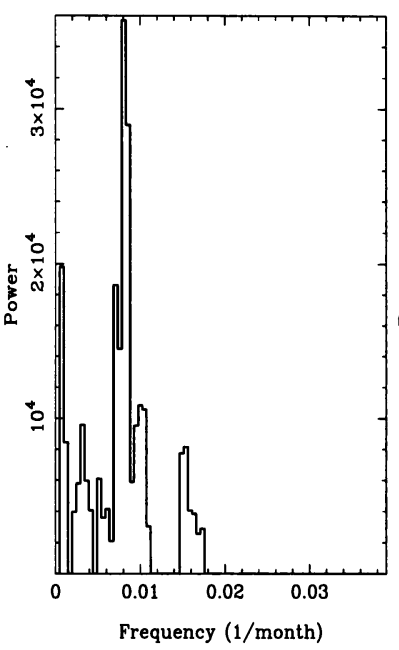

(c)

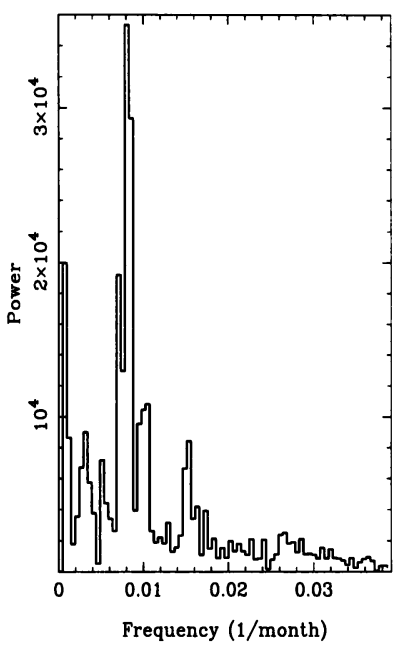

Figure 3. Fourier transform of the Wolf sunspot number (a), of its Fourier (b) and wavelet (c) reconstruction.

fit adequately represents the cloud of points. This has confirmed the model of sunspot occurrence which has been proposed by Morfill et al. (1991). Indeed, on observational and physical grounds, those authors have suggested that a Poisson distribution can mimic the behavior of the solar activity over times of the order of the solar rotation, which corresponds reasonably well to the month over which are averaged the daily sunspot numbers. We were thus entitled to use this parabolic fit in order to assess a likely "uncertainty" to any actual value of the monthly sunspot number according to the fitting relation.

We then proceed to the sunspot number analysis in a similar way as our previous work on the solar radius. The results show that the number of parameters retained for the wavelet and Fourier reconstruction is closer, for a reconstruction of the same quality (Vigouroux and Delache 1994). However, Fourier analysis needs a more severe criterion to reach this required quality.

Examination of the spectra of the original and reconstructed time series (figure 3 ) shows that all spectral estimates corresponding to frequencies below $(0.018 \text { month })^{-1}$ are preserved as significant. Both wavelet and Fourier analysis tell us that a large frequency band conveys significant informations. A purely modal description of the data as interferences between a few standing oscillations is therefore a dangerous biased selection of the actual information contained in the data. 


\section{Study of the total solar irradiance}

In collaboration with Judit Pap (from JPL and UCLA) we have made a detailed study of the total solar irradiance data set. We have investigated the real origins of "error bars" and their relation to the solar cycle (Pap et al. 1996). Plotting the irradiance error bars as a function of their corresponding value provides an interesting tool to separate the random fluctuations related to instrumental effects from real solar variability. Putting all the daily variability within 30-day into error bars calculated as dispersion allows again to study the cycle dependence of those dispersions. We showed that the dispersion values plots as function of their 30-day corresponding mean values may help in determining the length and precise dates for the solar cycle minimum. This study applied to other solar indices related to total irradiance (full disk integrated magnetic flux, $\mathrm{Mg}$ core-to-wing ratio, Photometric Sunspot Index which is an indicator of the irradiance deficit due to sunspots) has underlined some differences related to the minimum length between those time series.

Using several techniques (wavelet analysis to retrieve the solar cycle and then cross-correlation between scales of the wavelet transform of the analysis residual time series), we then found that the magnetic field and $\mathrm{Mg} \mathrm{c} / \mathrm{w}$ ratio (or total irradiance corrected from the sunspots darkening) have the same behavior but for time scales shorter than 8 months. It should be pointed out that those 8 months are related to the complex of activity evolution across the solar disk and that all of those three indices are related to them. We then found a correlation for 10 months delay between the total irradiance corrected from the sunspots darkening and the $\mathrm{Mg} \mathrm{c} / \mathrm{w}$ ratio when the latter is leading the former (Vigouroux and Pap 1995, Vigouroux et al. 1997). The observed time delays between various data sets representing photospheric and chromospheric conditions indicate that the response of the chromospheric layers to the magnetic field variations is quite different from that of the photosphere. The observations of some instruments on the SOlar Helioseismology Observatory (SOHO) satellite will dramatically improve our knowledge and capability in interpreting those results. Furthermore, analysis and interpretation of the time-delays found between the magnetic field and solar radiation emitted from different layers of the solar atmosphere will lead to a better understanding firstly of the dynamics taking place below, in, and above the photosphere and secondly of the basic mechanism governing the solar variability.

\section{Conclusion}

I am indebted to Philippe Delache for his help and for all he taught me. Although he was the director of the Observatoire de la Côte d'Azur at the 


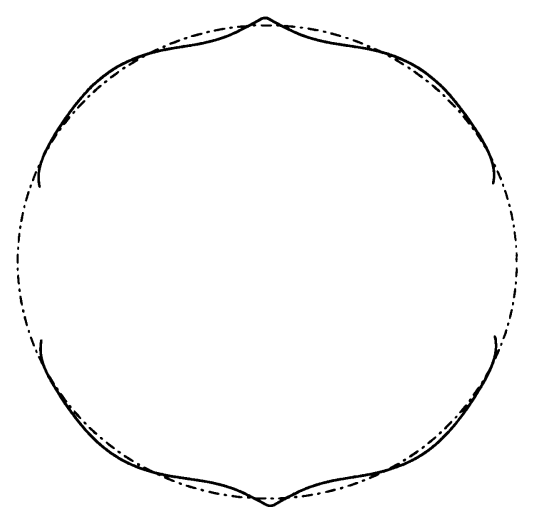

Figure 4. Perfect spherical Sun (dashed line) compared to the shape of the Sun as observed by F. Laclare (solid line).

time I begun my thesis, he nevertheless saved time for discussions and advice. He also always encouraged me to meet other scientists, to participate to colloquium and summer school, to present the work I did under his direction, instead of being sticked to the computer looking for a better solution to some problem. He introduced me into the international community.

I cannot end without speaking again of the radius. In section 2 , I mentioned that Francis Laclare spans a large range of heliographic latitudes thanks to his 11 prisms. It has appeared that the radius has not the same value whether it is measured near poles, near equator or near $45^{\circ}$ of heliographic latitude. Figure 4 shows a perfect circle representative of a perfect Sun and some distorted circle which is the actual shape of the Sun as measured by $\mathrm{F}$. Laclare (the deviations from the perfect circle have been multiplied by 500 in order to make them clear): I believe Philippe Delache would have loved to see the Sun as a lemon.

\section{References}

Delache, Ph., Laclare, F. and Sadsaoud, H. (1985) Long period oscillations in solar diameter measurements, Nature, 317, 416

Delache, Ph. (1988) Variability of the solar diameter, Adv. Space Res., 8, $\mathbf{n}^{\circ} \mathbf{7}, 119$

Delache, Ph., Laclare, F. and Sadsaoud, H. (1988) Long periods in diameter, irradiance and activity of the Sun, in Advances in helio-asteroseismology, eds. J. ChristensenDalsgaard et S. Frandsen, pp. 223

Delache, Ph., Gavryusev, V., Gavryuseva, E., Laclare, F., Régulo, C. and Roca Cortés, T. (1993) Time correlation between solar structural parameters: $p$-mode frequencies, radius, and neutrino flux, Astrophys. $J, 407,801$

Gavryusev, V., Gavryuseva, E., Delache, Ph. and Laclare, F. (1994) Periodicities in solar radius measurements, Astron. Astrophys., 286, 305 
Laclare, F. (1983) Mesures du diamètre solaire à l'astrolabe, Astron. Astrophys., 125, 200

Laclare, F., Delmas, C., Coin, J.P. and Irbah, A. (1996) Measurements and variations of the solar diameter, Solar Physics, 166, 211

Leister, N.V. and Benevides-Soares, P. (1990) Variations du diamètre solaire, C.R. Acad. Sci. Paris, t. 311, Série II, 399

Morfill, G. E., Scheingraber, H., Voges, W. and Sonett, C. P. (1991) Sunspot number variations: stochastic or chaotic, in The Sun in time, eds. C.P. Sonett, M.S. Giampapa et M.S. Matthews, University of Arizona Press, Tucson, pp. 30

Pap, J. M., Vigouroux, A. and Delache, Ph. (1996) Study of the distribution of daily fluctuations in observed solar irradiances and other full disk indices of solar activity, Solar Physics, 167, 125

Ribes, E., Ribes, J. C., Vince, I. et Merlin, Ph. (1988) A survey of historical and recent solar diameter observations, Adv. Space Res., 8, 129

Spruit, H.C. (1994) Theories of radius and luminosity variations, in The solar engine and its influence on terrestrial atmosphere and climate, ed. E. Nesme-Ribes, NATO A.S.I. Series 25, Springer-Verlag Berlin Heidelberg, pp. 107

Vigouroux, A. and Delache, Ph. (1993) Fourier versus wavelet analysis of solar diameter variability, Astron. Astrophys., 278, 607

Vigouroux, A. and Delache, Ph. (1994) Sunspot numbers uncertainties and parametric representations of solar activity variations, Solar Physics, 152, 267

Vigouroux, A. and Pap, J. M. (1995) Studying solar irradiance variability with wavelet technique, in Solar drivers of interplanetary and terrestrial disturbances, A.S.P. Conference Series 95, eds. K.S. Balasubramaniam, S.L. Keil et R.N. Smartt, pp. 586

Vigouroux, A., Pap, J. M. and Delache, Ph. (1997) Estimating long-term solar irradiance variability: a new approach, Solar Physics, in press 